\title{
Reliability and Usefulness of the 30-15 Intermittent Fitness Test in Male and Female Professional Futsal Players
}

\author{
by \\ Santiago Valladares-Rodríguez, Ezequiel Rey¹, Marcos Mecías-Calvo², \\ Roberto Barcala-Furelos ${ }^{1}$, Antonio J. Bores-Cerezal ${ }^{2,3}$
}

The purpose of this study was to determine the reliability and usefulness of the 30-15 Intermittent Fitness Test (30-15IFT) in professional male and female futsal players. Thirteen male $(24.4 \pm 5.6$ years; $174.5 \pm 10.3 \mathrm{~cm} ; 70.3 \pm 9.9$ $\mathrm{kg})$ and fourteen female $(23.3 \pm 4.5$ years; $165.8 \pm 6.2 \mathrm{~cm} ; 61.7 \pm 5.5 \mathrm{~kg})$ professional futsal players performed the 30$15 I F T$ on two occasions, separated by 5 days. Maximal intermittent running velocity (VIFT) and heart rate at exhaustion (HRpeak) data were collected for both tests. Reliability was assessed by the intraclass correlation coefficient (ICC), typical error (TE) expressed as a coefficient of variation (CV), and smallest worthwhile change (SWC). VIFT demonstrated very good reliability between sessions, both for male $(I C C=0.92)$ and female $(I C C=0.96)$ players. As the TE for VIFT and HRpeak was similar to the calculated SWC for both male and female players, the usefulness of the test was rated as "medium". A change in performance of at least 2 stages in male players, or a change of more than 1 stage in female players could be interpreted as a meaningful change in aerobic futsal fitness. The results of this study demonstrate that the 30-15IFT is both a reliable and useful test for male and female professional futsal players.

Key words: aerobic, exercise performance, exercise physiology, physical fitness, training.

\section{Introduction}

Futsal is a variation of soccer played on a $40 \times 20 \mathrm{~m}$ (length per width, respectively) indoor court with $3 \times 2 \mathrm{~m}$ goals. It started in South America in 1930 and has become a popular indoor sport worldwide since then. Futsal is a team sport played between two teams with 4 outfield players (defender, winger and pivot) and one goalkeeper; another important aspect is that unlimited substitutions are permitted during the game (De Moura et al., 2013).

Despite its popularity and competitiveness, as it is a relatively recent sport, research on futsal is still scarce, even more so if compared to the sport it originated from, i.e., soccer. Match analysis and physiological monitoring has shown that futsal may be characterized as an intermittent high-intensity sport (Barbero-Álvarez et al., 2009; Dogramaci et al., 2011) with frequent occurrence of short sprints (Caetano et al., 2015). Time-motion analysis studies have demonstrated that professional futsal players spend $>22 \%$ of total match time undertaking high-intensity running and sprinting activities $\left(>18.1 \mathrm{~km} \cdot \mathrm{h}^{-1}\right)$ that are typically in the form of short bursts (Barbero-Álvarez et al., 2008). Analysis of the heart rate (HR), as an indicator of the internal load, has confirmed an elevated intensity during official matches, thus demonstrating that the players, during most of the time, maintain intensity of effort at $85 \%$ of the maximum heart rate (Barbero-Álvarez et al., 2008; Castagna et al., 2009; Rodrigues et al., 2011). Moreover, direct game $\mathrm{VO}_{2}$ assessment showed that ahletes needed at least $50-55 \mathrm{ml} \cdot \mathrm{kg}^{-1} \cdot \mathrm{min}^{-1}$

1 - Faculty of Education and Sports Sciences, University of Vigo, Pontevedra, Spain.

2 - European University of Atlantic, Santander, Spain.

3 - Physical Trainer Spain National Futsal Team, The Royal Spanish Football Federation. 
of $\mathrm{VO}_{2 \text { peak }}$ to play futsal at the professional level (Castagna et al., 2009). Collectively, these findings highlight that futsal players require high levels of aerobic power that should be carefully considered in talent selection and training at the professional level (Galy et al., 2015). The importance of aerobic capacity in futsal is evidenced by its ability to discriminate between players of different levels of performance (Castagna et al., 2009; Pedro et al., 2014). Consequently, in order to prescribe individualized training programs, futsal players' cardiorespiratory fitness should be assessed accordingly.

Incremental laboratory-based protocols using running treadmills and cycle ergometers have commonly been used to asses cardiorespiratory fitness. However, these tests are time consuming, require the use of expensive equipment, and may cause disruption of the usual training schedule as they require players to make several visits to the laboratory. Consequently, indirect methods emerged as alternatives to estimate $\mathrm{VO}_{2 \text { peak }}$ in a single incremental test.

Recently, the 30-15 Intermittent Fitness Test (30-15IFT) has been introduced as a practical alternative to the commonly used field tests in team-sport athletes (Buchheit, 2008). The 30-15IFT is an intermittent incremental shuttle-run test that is designed to elicit the peak heart rate (HRpeak) and $\mathrm{VO}_{2 \text { peak, }}$ in addition to providing an overall measure of sport-specific fitness (Buchheit, 2008). The validity and reliability of the 30-15IFT has recently been demonstrated among soccer, rugby, basketball, handball, and ice hockey cohorts (Buchheit, 2008; Buchheit et al., 2011; Scott et al., 2015; Thomas et al., 2015). What makes this test appealing to practitioners is its validity in the individual prescription of high-intensity interval training (HIIT), using the final running velocity reached at the end of the 30-15IFT (VIFT) (Buchheit, 2008), overcoming the limitations of other field-based tests. As such, prescribing training based on VIFT seems to increase the homogeneity across the playing group and further standardize training content at a team level (Buchheit, 2008). The VIFT helps players with different physiological profiles to reach similar cardiorespiratory levels so that interindividual differences become less important compared to other continuous linear or shuttle run tests (Buchheit, 2008). Moreover, the 30-15IFT has been perceived to be less "taxing" than continuous field-based tests in $70 \%$ of team sport athletes examined (Buchheit, 2008). In addition, different publications have proven the usefulness of the 30-15IFT, that is, its ability to monitor changes in aerobic fitness after training programs via measurement of direct test performance or indirect markers such as submaximal HRs (Buchheit et al., 2011; Scott et al., 2015; Thomas et al., 2015).

An appropriate method for assessing usefulness is via the smallest worthwhile change (SWC) so that one may determine if a real change has occurred over time for subsequent testing periods. Furthermore, reliability data allow one to calculate the minimal individual change that can be interpreted as real with an acceptable probability level, that is, the minimal detectable change (MD). Consideration of the MD of a particular test is of great interest for monitoring the progress of athletes as intertrial variation may incorrectly suggest a change that has not exceeded the threshold of error (Hopkins, 2000).

Despite the specificity of this test for the evaluation of team sports athletes, no researchers have investigated the reliability and usefulness of the 30-15IFT in futsal players. Therefore, the aim of this study was to determine between-session reliability (including both VIFT and HRpeak) of the $30-15$ IFT in professional male and female futsal players.

\section{Methods}

Design

A within-subject repeated measures research design was used to determine the reliability of the 30-15IFт performed during two separate testing sessions, 5 days apart. To standardize the training state, the two testing sessions were performed at the end of the preseason period before the competitive season. It was considered that examining professional futsal players during their actual training period would increase the relevance and applicability of the results. All subjects were familiar with running patterns and audio signals of the test. Because of the specificity of the test and subsequent conditioning prescription, during testing sessions the participants were required to wear the same athletic equipment and measurements were conducted at the same time of day to minimize 
the effect of diurnal variations on the selected variables. Tests were performed on an indoor synthetic-floor court. To avoid undue fatigue before testing, players refrained from heavy training for the preceding 24 hours. Moreover, to reduce the influence of uncontrolled variables, all futsal players were instructed to maintain their habitual lifestyle and normal dietary habits (fluid and food intake) before and during the study. Additionally, before each testing session, players complied with the following pre-test guidelines: (a) not to consume any energy/performanceenhancing drinks or supplements $48 \mathrm{~h}$ before testing; (b) not to take beverages containing caffeine or alcohol at least three hours before testing; and (c) not to consume food at least $2 \mathrm{~h}$ before testing. All testing sessions were performed on an indoor court under stable environmental conditions (ambient temperature: $19-24^{\circ} \mathrm{C}$ and humidity: $\left.50-65 \%\right)$. Reliability of the 30-15 IFт was examined using the intraclass correlation coefficient (ICC) as well as the typical error of measurement (TE), expressed as a coefficient of variation $(\% \mathrm{CV})$, similarly to the previously reported methods (Buchheit et al., 2011; Scott et al., 2015; Thomas et al., 2015).

\section{Participants}

The subject pool $(n=27)$ was comprised of male professional futsal players competing in Spanish First Division Professional Futsal League (Liga Nacional de Fútbol Sala, LNFS) $(n=13 ; 24.4$ \pm 5.6 years; $174.5 \pm 10.3 \mathrm{~cm} ; 70.3 \pm 9.9 \mathrm{~kg}$ ) and female professional futsal players competing in the Spanish Female First Division $(n=14 ; 23.3 \pm$ 4.5 years; $165.8 \pm 6.2 \mathrm{~cm} ; 61.7 \pm 5.5 \mathrm{~kg}$ ). Male players were involved in $7.9 \pm 0.5 \mathrm{~h} \cdot \mathrm{wk}^{-1}$ of physical training, whereas female players completed $8.1 \pm 0.7 \mathrm{~h} \cdot \mathrm{wk}^{-1}$. All players provided written informed consent before participation and were cleared of injury by the team's medical staff before completing the testing sessions. The study conformed to the principles of the World Medical Association's Declaration of Helsinki and was approved by the Institutional Review Board for the use of Human Subjects of the Department of Physical Education and Sport Sciences at the University of Vigo.

\section{Measures}

The 30-15 Intermittent Fitness Test. The 3015 IFT was performed as previously described (Buchheit, 2008) on an indoor synthetic-floor court. The $30-15$ IFT consisted of $30 \mathrm{~s}$ shuttle runs interspersed with $15 \mathrm{~s}$ periods of passive recovery. The initial running velocity was set at $8 \mathrm{~km} \cdot \mathrm{h}^{-1}$ for the first $30 \mathrm{~s}$ run and increased by $0.5 \mathrm{~km} \cdot \mathrm{h}^{-1}$ with every subsequent $45 \mathrm{~s}$ stage. Players ran back and forth between 2 lines set $40 \mathrm{~m}$ apart at a pace governed by a pre-recorded beep. This pacing strategy allowed subjects to run at appropriate intervals and helped them adjust their running speed as when they entered $3 \mathrm{~m}$ zones at each end, as well as reached the middle zone $(20 \mathrm{~m}$ line), a short beep was emitted (Figure 1). During the $15 \mathrm{~s}$ recovery period, each player walked forward to the closest of the 3 lines (at the middle or at one of the ends of the running area, depending on where the previous stage was completed), in preparation for the next stage. Players were instructed to complete as many stages as possible, and the test ended when a player could no longer maintain the imposed running speed or when they were unable to reach a $3 \mathrm{~m}$ zone around each line at the moment of emitting the audio signal on 3 consecutive occasions. If players were unable to complete the stage, then their score was recorded as the stage that they last completed successfully, and the running velocity was registered as their maximal 30-15 IFT running velocity (VIFT) (Buchheit, 2008).

Heart Rate Measurement. The heart rate (HR) was recorded using a Polar $\mathrm{T}^{2}$ system with R-R technology (Polar Electro Oy, Kempele, Finland). The HR was continually recorded at 1 $\mathrm{Hz}$ throughout each 30-15 IFT. Peak HR (HR peak) was recorded as the highest HR registered during the final $30 \mathrm{~s}$ of the test.

\section{Statistical Analysis}

The distribution of each variable was examined using the Shapiro-Wilk normality tests, and homogeneity of variance was verified with a Levene's test using the statistical package SPSS for Macintosh (version 21.0, Chicago, IL, USA). Data are presented as either means $\pm S D$ or means with $90 \%$ confidence intervals $(90 \%$ CI $)$ where specified. To examine the reliability of the test over the 2 consecutive trials, paired sample $t$-tests were used to identify any significant differences between test-retest data. The magnitude of difference between consecutive trials was also expressed as standardized mean differences (Cohen effect sizes [ES]). Effect sizes with values of $0.2,0.5$, and 0.8 were considered to represent 
small, medium, and large differences, respectively (Cohen, 1988). Change in mean between trials, ICC, and TE, expressed as a coefficient of variation $(\mathrm{CV})$, were calculated through Hopkins available on-line spreadsheet (Hopkins, 2000). As a criterion to declare the reliable variable, the $\mathrm{CV}$ was set at $<5 \%$ (Hopkins, 2004). To assess meaningful changes in the fitness level or performance, Hopkins (2015) had previously proposed that the smallest worthwhile change (SWC) was significant in the assessment of performance markers due to the noise associated with physical testing. Therefore, the usefulness of the test was assessed by comparing the SWC in $\mathrm{V}_{\mathrm{IFT}}$ and HReak (SWC; 0.2 multiplied by the between-subject $S D$, based on the Cohen's effect size principle) with $\mathrm{TE}$ of these measures (Hopkins et al., 2001). In line with previous research, if the TE was higher than the SWC, the evaluation of test was "marginal", if the TE was similar to the SWC, the evaluation was "medium"; and if the TE was less than the SWC, an evaluation of "good" was given to the test (Buchheit, 2008; Scott et al., 2015). The smallest difference needed to be considered real (MD, corresponding to a change likely to be almost certain) was calculated as the TE $\times 1.96 \times \sqrt{ } 2$ (Weir, 2005). It can be interpreted as the minimal change that is required in a test performance before a coach can be confident that a real change has occurred (e.g., post-training phase).

\section{Results}

\section{Reliability}

Values for $\mathrm{V}_{\text {IFT }}$ and $\mathrm{HR}_{\text {peak }}$ were $20.2 \pm 1.7$ $\mathrm{km} \cdot \mathrm{h}^{-1}$ and $189 \pm 7 \mathrm{~b} \cdot \mathrm{min}^{-1}$ for the first test, and $20.3 \pm 1.6 \mathrm{~km} \cdot \mathrm{h}^{-1}$ and $188 \pm 6 \mathrm{~b} \cdot \mathrm{min}^{-1}$ for the second test in male futsal players. In female players the values were $17.4 \pm 1.3 \mathrm{~km} \cdot \mathrm{h}^{-1}$ and $192 \pm 7 \mathrm{~b} \cdot \mathrm{min}^{-1}$ for the first test, and $17.3 \pm 1.2 \mathrm{~km} \cdot \mathrm{h}^{-1}$ and $190 \pm 6$ $\mathrm{b} \cdot \mathrm{min}^{-1}$ for the second test, respectively. The pairwise analysis revealed no significant differences between the 2 trials for all variables $(p$ $>$ 0.05). Differences in all variables between repeated trials displayed "trivial" ES. ICC values were very high both for male (0.92) and female (0.96) futsal players, whereas $\mathrm{CV}$ values were low (1.4-1.5\%).

\section{Usefulness}

TE of measurement values for $\mathrm{V}_{\text {IFT }}$ and $H R_{\text {peak }}$ was similar to the calculated SWC for both male and female futsal players, thus the usefulness of the test was rated as "medium" for these variables (Table 1). MD values are also presented in Table 1.

\begin{tabular}{|rcccc|}
\hline & & & Table 1 \\
Measures of reliability for maximal intermittent running velocity (VIFT) \\
and the peak heart rate $\left(\mathrm{HR}_{\text {peak }}\right)$ during the 30-15 Intermittent Fitness Test.
\end{tabular}




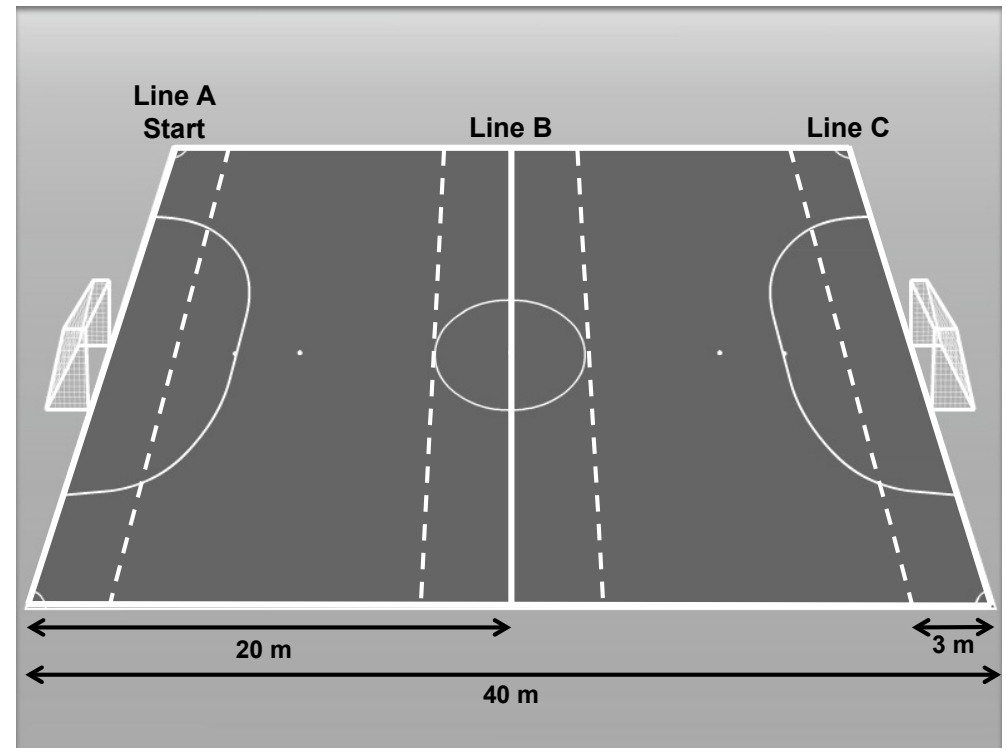

Figure 1

Court setup of the 30-15 Intermittent Fitness Test.

\section{Discussion}

One of the keys to success in sports training includes control of training loads and their effects on physiological variables. Consequently, sports technicians seek and develop tools and protocols that can be useful and applicable to their sport. In this way, the 30-15IFT has been developed as an intermittent incremental shuttle run test that demonstrates high validity and reliability in different team sports (Buchheit, 2005; Buchheit et al., 2011; Scott et al., 2015; Thomas et al., 2015). However, despite the specificity of this test to team-sport athletes, no study has previously investigated the reliability and usefulness of the 30-15IFT in professional futsal players. In agreement with previous research (Buchheit, 2005; Buchheit et al., 2011; Scott et al., 2015; Thomas et al., 2015), our results suggest that the 30-15IFT is a reliable method of assessing futsal player's maximal aerobic power. VIFT and HRpeak obtained during the test showed good reliability in both male and female professional futsal players. The usefulness of the test, that is, its ability to monitor a change in performance, was confirmed for male and female professional futsal players. Collectively, based on the current findings, sport scientists and coaches can consider the 30-15IFT to test futsal players in an ecologically valid environment.

The professional level of the tested subjects is considered a strength of the study. Overall, the results of the current study show that values of VIFT in elite futsal players were higher than those previously reported in young rugby players (Scott et al., 2015), as well as youth basketball and handball players (Buchheit, 2008).

The lack of differences between test-retest trials (i.e. $\mathrm{ES}=0.08-0.12$ ), indicating the absence of a learning effect, and the very high ICC values (i.e., 0.92-0.96), demonstrated that the 30-15IFT was a reliable tool to evaluate aerobic fitness of professional futsal players. The reliability of a test 
depends on a number of factors, such as the number of subjects, the number of performed trials, subject's skills, and motivation to perform well (Hopkins, 2000). Actually, the high skill level of futsal players who competed at the highest Spanish level and their high motivation to perform at their best during the testing sessions could have contributed to the high reliability of the findings. Thus, future research is strongly needed to verify whether this test could be applied also to futsal players of different levels.

The CV of the present study for male (CV $=1.5 \%)$ and female $(\mathrm{CV}=1.5 \%)$ futsal players was similar to that reported previously for handball $(\mathrm{CV}=1.7 \%)$, ice hockey $(\mathrm{CV}=1.6 \%)$, and soccer $(\mathrm{CV}=2.5 \%)$ cohorts (Buchheit, 2005; Buchheit et al., 2011; Scott et al., 2015; Thomas et al., 2015). Additionally, this result is comparable with findings previously reported in similar fieldbased tests in soccer players such as the Yo-Yo IR1 and Yo-Yo IR2 (Krustrup and Bangsbo, 2001; Krustrup et al., 2006). Furthermore, the reliability of HR measures $(\mathrm{CV}=1.4 \%)$ is in accordance with previous research that has examined the reliability of HRpeak during a modified 30-15IFT on ice hockey $(\mathrm{CV}=0.7 \%)$ and in rugby players $(\mathrm{CV}=0.6 \%)$ (Buchheit et al., 2001; Scott et al., 2015). The good reliability of the HRpeak found in the present study (ICC $=0.90$ for males; ICC $=0.89$ for females), enables the use of this indirect measure as an important parameter to control the internal training load during functional and generic training sessions designed to develop aerobic fitness in futsal players. Additionally, the HRpeak can be used by coaches in conjunction with VIFT in the prescription of HIIT models, as well as to monitor performance improvements induced during the competitive season (da Silva et al., 2015).

A secondary purpose of this research was to determine the usefulness of the 30-15IFT among professional male and female futsal players. Different studies recommend extending analyses of absolute reliability by the use of the SWC (Buchheit et al., 2011; Thomas et al., 2015). The SWC is defined as the minimal individual change that can be interpreted as real with an acceptable probability level (Buchheit et al., 2011; Thomas et al., 2015). In this context, we also calculated the likelihood that the true value of estimated difference in the 30-15IFT test performance was substantial (i.e. larger than the $\mathrm{SWC}$ ). Since the TE value was not higher than that calculated for the SWC, the usefulness (the ability of the test to detect changes in cardiorespiratory fitness in male and female futsal players) of the test was rated as "medium" for VIFT and HRpeak (Table 1). Therefore, from a practical point of view, taking into account the MD results, we suggest that a change of at least 2 stages $\left(>1 \mathrm{~km} \cdot \mathrm{h}^{-}\right.$ 1) for male and 1 stage for female $\left(>0.5 \mathrm{~km} \cdot \mathrm{h}^{-1}\right)$ futsal players in VIFT could be considered substantial or "real." The results of this study support previous findings (Buchheit et al., 2011; Thomas et al., 2015). The aforementioned threshold can be used by sports scientists to monitor meaningful improvement in 30-15IFT performance in professional futsal players. Additionally, the usefulness of the HRpeak taken from the tests was rated as "medium" (Table 1). The usefulness values found in this study have important implications in sport settings as they enable strength and conditioning coaches to make knowledgeable decisions regarding whether a real change has occurred between testing sessions after application of a training program (i.e. HIIT protocols) or whether the observed change is simply a product of typical error. A previous study confirmed the validity of the HRpeak to identify training adaptations in other team sports (da Silva et al., 2015). Those authors observed a significant decrease in the HRpeak at the end of the third week of two interval training programs in soccer players, that supports the usefulness of the HRpeak to control the internal load during HIIT training sessions.

The interpretation and broader implications of the current data must be undertaken within the limits of the specific data collection undertaken. While the study had many unique aspects (elite level players, male and female players), there were some limitations that should be noted. First of all, although the number of participants in this study was similar to these reported in other studies that had assessed reliability of the 30-15IFT, our sample size was relatively small. The second limitation is the fact that ergoespirometric analysis was not included in an attempt to keep the study simple, noninvasive, and practical. Despite these limitations, this study provides normative performance and training data for elite futsal players, thus coaches 
along with strength and conditioning specialists could use this information to determine performance standards.

The implementation of field fitness test batteries in the training assessment routine is a time consuming practice and their usefulness for training prescription and performance control highly depends on the validity and reliability of the used item. The most important findings of the present study indicate that the 30-15IFT is a reliable protocol for cardiorespiratory fitness assessment in male and female futsal athletes. The test is inexpensive, it can simultaneously evaluate an entire futsal squad, it can be completed within 30 minutes, and it requires little equipment and few resources. The 30-15IFT can be routinely included by sports scientists and strength and conditioning coaches in an assessment battery for futsal players. We suggest that in professional futsal players, coaches may interpret a performance improvement of at least 2 stages in males and 1 stage in females, for example after a training program, as an "almost certain" increase in cardiorespiratory performance.

The VIFT may guide futsal fitness coaches in aerobic training prescription when using generic training such as running HIIT (Buchheit and Laursen, 2013), as well as to monitor performance changes induced throughout the season.

To our knowledge, this is the first time that the 30-15IFT has been applied in male and female professional futsal players. This study contributes to the literature on the physiological profile of futsal players, especially as far as the female futsal population is concerned. In addition, this study provides normative data for professional futsal players of VIFT, so that conditioning coaches can use this information to determine standards of aerobic performance during the in-season period. Further studies addressing male and female futsal players of different playing levels are warranted.

\section{References}

Álvarez JCB, D'Ottavio S, Vera JG, Castagna C. Aerobic fitness in futsal players of different competitive level. J Strength Cond Res, 2009; 23: 2163-2166

Barbero-Álvarez JC, Soto VM, Barbero-Alvarez V, Granda-Vera J. Match analysis and heart rate of futsal players during competition. J Sports Sci, 2008; 26: 63-73

Buchheit M. The 30-15 intermittent fitness test: Reliability and implication for interval training of intermittent team sport players [abstract no. 1231]. 10th European Congress of Sport Science, Belgrade, July 13-16; 2005

Buchheit M. The 30-15 intermittent fitness test: accuracy for individualizing interval training of young intermittent sport players. J Strength Cond Res, 2008; 22: 365-374

Buchheit M, Laursen PB High-Intensity interval training, solutions to the programming puzzle. Part II: anaerobic energy, neuromuscular load and practical applications. Sports Med, 2013; 43: 927-954

Buchheit M, Lefebvre B, Laursen PB, Ahmaidi S. Reliability, usefulness, and validity of the 30-15 Intermittent Ice Test in young elite ice hockey players. J Strength Cond Res, 2001; 25: 1457-1464

Caetano FG, de Oliveira MJ, Marche AL, Nakamura FY, Cunha SA, Moura FA. Characterization of the sprint and repeated-sprint sequences performed by professional futsal players, according to playing position, during official matches. J Appl Biomech, 2015; 31: 423-429

Castagna C, D'Ottavio S, Vera JG, Álvarez JCB. Match demands of professional Futsal: A case study. J Sci Med Sport 2009; 12: 490-494

Cohen J. Statistical power analysis for the behavioural sciences (2nd ed). Hillsdale, NJ: Lawrence Erlbaum; 1988

De Moura NR, Borges LS, Santos VC, Joel GB, Bortolon JR, Hirabara SM, Cury-Boaventura MF, Pithon-Curi TC, Curi R, Hatanaka E. Muscle lesions and inflammation in futsal players according to their tactical positions. J Strength Cond Res, 2013; 27: 2612-2618

Dogramaci SN, Watsford ML, Murphy AJ. Time-motion analysis of international and national level futsal. J Strength Cond Res, 2011; 25: 646-651

da Silva JF, Nakamura, FY, Carminatti, LJ, Dittrich, N, Cetolin, T, Guglielmo, LGA. The effect of two generic 
aerobic interval training methods on laboratory and field test performance in soccer players. J Strength Cond Res, 2015; 29: 1666-1672

Galy O, Zongo P, Chamari K, Chaouachi A, Michalak E, Dellal A, Castagna C, Hue O. Anthropometric and physiological characteristics of Melanesian futsal players: a first approach to talent identification in Oceania. Biol Sport, 2015; 32: 135-141

Hopkins WG. Measures of reliability in sports medicine and science. Sports Med, 2000; 30: 1-15

Hopkins WG. How to interpret changes in an athletic performance test. Sports Sci, 2004; 8: 1-7

Hopkins WG. Reliability from consecutive pairs of trials (Excel spreadsheet). A new view of statistics sportssciorg: Internet Society for Sport Science. Available at: sportsciorg/resource/statsxrelyxls; accessed 19.08.2015

Hopkins WG, Schabort EJ, Hawley JA. Reliability of power in physical performance tests. Sports Med, 2001; 31: 211-234

Krustrup P, Bangsbo J. Physiological demands of top-class soccer refereeing in relation to physical capacity: Effect of intense intermittent exercise training. J Sports Sci, 2001; 19: 881-891

Krustrup P, Mohr M, Nybo L, Jensen JM, Nielsen JJ, Bangsbo J. The Yo-Yo IR2 test: Physiological response, reliability, and application to elite soccer. Med Sci Sports Exerc, 2006; 38: 1666-1673

Pedro RE, Milanez VF, Boullosa DA, Nakamura FY. Running speeds at ventilatory threshold and maximal oxygen consumption discriminate futsal competitive level. J Strength Cond Res, 2014; 27: 514-518

Rodrigues VM, Ramos GP, Mendes TT, Cabido CET, Melo ES, Condessa LA, Coelho D, Garcia ES. Intensity of official Futsal matches. J Strength Cond Res, 2011; 25: 2482-2487

Scott TJ, Delaney JA, Duthie GM, Sanctuary CE, Ballard DA, Hickmans JA, Dascombe BJ. Reliability and usefulness of the 30-15 intermittent fitness test in rugby league. J Strength Cond Res, 2015; 29: 19851990

Thomas C, Dos'Santos T, Jones PA, Comfort P. Reliability of the 30-15 Intermittent Fitness Test in semiprofessional soccer players. Int J Sports Physiol Perform, doi:10.1123/ijspp.2015-0056

Weir JP. Quantifying test-retest reliability using the intraclass correlation coefficient and the SEM. J Strength Cond Res, 2005; 19: 231-240

\section{Corresponding author:}

\section{Ezequiel Rey, PhD}

Faculty of Education and Sports Sciences, University of Vigo,

Campus Universitario s/n, 36005, Pontevedra, Spain

Phone: + 349868001700

E-mail: zequirey@uvigo.es 